\title{
Simvastatin inhibits the expression of stemness-related genes and the metastatic invasion of human cancer cells via destruction of the cytoskeleton
}

\author{
ROSARITA TATË ${ }^{1 *}$, ENRICA ZONA $^{1 *}$, ROSANNA DE CICCO ${ }^{1}$, \\ VERONICA TROTTA ${ }^{1}$, MARIA URCIUOLI ${ }^{2}$, ALESSANDRO MORELLI ${ }^{1}$, SALVATORE BAIANO ${ }^{1}$, \\ ROSA CARNUCCIO ${ }^{3}$, MARIA PIA FUGGETTA ${ }^{4}$ and FRANCO MORELLI ${ }^{1}$ \\ ${ }^{1}$ Institute of Genetics and Biophysics Andriano Buzzati Traverso, CNR, Naples; \\ ${ }^{2}$ Department of Medical Clinic, Second University of Naples, Naples; ${ }^{3}$ Department of Pharmacy, \\ University of Naples Federico II, Naples; ${ }^{4}$ Institute of Translational Pharmacology, CNR, Rome, Italy
}

Received June 14, 2017; Accepted September 15, 2017

DOI: $10.3892 /$ ijo. 2017.4158

\begin{abstract}
Statins are a class of drugs that inhibit the ratelimiting steps in the cholesterol biosynthesis pathway. They act by inhibiting 3-hydroxy-3-methylglutaryl CoA (HMG-CoA) reductase, which catalyzes the conversion of $\mathrm{HMG}-\mathrm{CoA}$ to mevalonate. Blocking of mevalonate synthesis leads to inhibition of the farnesylation and geranylgeranylation of several functional proteins, such as RhoA and other small guanosine triphosphate-binding proteins, that are important in maintaining the undifferentiated status of the cells. In the present study, we hypothesized that simvastatin, likely through the inhibition of farnesylation and geranylgeranylation of Rac1, $\mathrm{Cd} 42$ and RhoA, induces a destruction/restructuration of the cytoskeleton that decreases mechanical strain transfer to the nuclei, inducing the loss of transmission of regulatory signals from the cytoskeleton to the nucleoskeleton. Although this remains at present a hypothesis and is not easy to define if the de-structuration of the cytoskeleton is a secondary effect of simvastatin treatment or the inhibition of post-translational protein modification have a precise role in the structuration of actin cytoskeleton, we speculate that these signal variations could inhibit the expression of certain stemness genes, which could therefore be considered nucleoskeleton-associated and mechanically regulated genes. On the other hand, the restructuration of the cytoskeleton inhibits the formation of lamellipodia and filopodia, which likely decreases the
\end{abstract}

Correspondence to: Professor Franco Morelli, Institute of Genetics and Biophysics Andriano Buzzati Traverso, CNR, Via Pietro Castellino 111, I-80131 Naples, Italy

E-mail: morelli@igb.cnr.it

${ }^{*}$ Contributed equally

Key words: simvastatin, cytoskeleton destruction, stemness genes, RhoA geranylgeranylation capability of cancer cells to invade the extracellular matrix, thereby modulating the equilibrium between proliferation, differentiation and metastatic invasion in human cancer cells. On the basis of our results we think that simvastatin, alone or in combination with conventional drugs, may have a possible role in cancer therapy.

\section{Introduction}

Statins, also known as 3-hydroxy-3-methylglutaryl coenzyme A (HMG-CoA) reductase inhibitors, are a class of drugs that inhibit the rate-limiting steps in the cholesterol biosynthesis pathway $(1,2)$. Any compound leading to the depletion of cholesterol affects various cellular events, and also impairs homeostasis and cholesterol-independent pleiotropic mechanisms, which are likely a consequence of blocking intracellular signaling (3).

Statins, as described in the schematic diagram of the biochemical pathway in Fig. 1, act by inhibiting HMG-CoA reductase and therefore the conversion of HMG-CoA to mevalonate (4). Blocking mevalonate synthesis leads to the inhibition of farnesyl and geranylgeranyl modifications of several functional proteins, such as RhoA, a small guanosine triphosphate (GTP)-binding protein. In particular, simvastatin selectively blocks RhoA geranylgeranylation and its translocation to the cell membrane, where it interacts with downstream effectors to control cell cycle progression (5). Rho activation by geranylgeranylation leads to a reduction in the inhibitory phosphorylation of YAP/TAZ, and thus sustains YAP/TAZ nuclear accumulation (6), thereby serving a fundamental role in the control of tissue proliferation and organ growth, as well as tumor onset and progression.

Considering the role of Rho, any inhibition of its synthesis or modification may induce a reduction in proliferation and thereby suppress tumor onset. Furthermore, tumors contain a subset of cancer stem cells that drive metastatic spread and Rho function may be associated with cancer stem cells. In these cells, genes that confer the characteristics of multipotency to the cells, associated with hyperproliferation and blocking of 


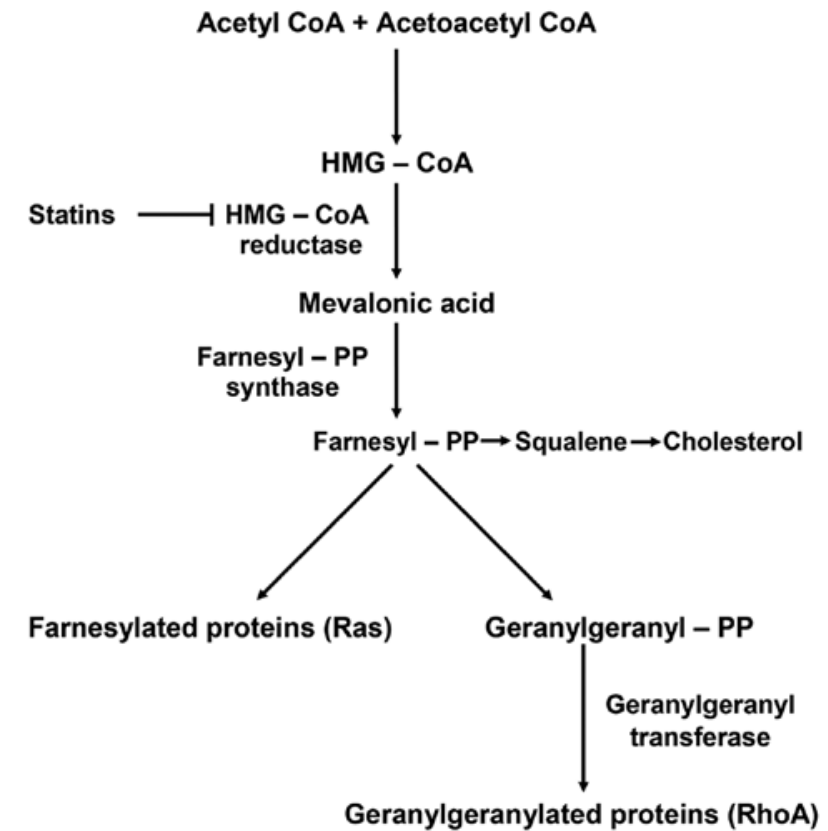

Figure 1. Schematic diagram of the mevalonate pathway. The figure shows the mevalonate pathway and the associated reactions of farnesylation and geranylgeranylation.

differentiation, are overexpressed or re-expressed. Although several genes are considered to contribute to maintaining hyperproliferative status, we have focused on three representative stemness-related genes, Oct4, Sox 2 and Nanog (7-9), with the aim of investigating whether simvastatin is able to modify, directly or indirectly, the expression equilibrium of these genes or influence the translation process of the respective proteins.

Oct 4 , Sox 2 and Nanog are transcription factors that regulate the pluripotency of human pluripotent stem cells (hPSCs) and regulate mesendoderm and ectoderm differentiation (10-15). It has been clearly demonstrated that, in embryonic stem cells (ESCs), Sox 2 interacts with Oct4 to form a regulatory dimer complex (16-21), indicating that Sox 2 is positively auto-regulated by the Sox2-Oct4 complex. In human and mouse ESCs, Oct 4 , Sox 2 and Nanog form transcriptional regulatory circuitry to activate the expression of pluripotency-related genes and repress the expression of differentiation-related genes, and are implicated in tumorigenesis in various organs (22-39). Recently, it has been demonstrated that the inhibition of Sox 2 can induce the repression of YAP, TAZ and TEAD functions, exerting an oncosuppressive effect (40-45).

In tumorigenesis, the epithelial-to-mesenchymal transition (EMT) is an important developmental process in which immotile epithelial cells acquire mesenchymal features (46-49), along with the ability to spread through the extracellular matrix and surrounding tissues. EMT can be induced by several signal pathways, and principally by Wnt/ $\beta$-catenin (50-53). Sox 2 and $\beta$-catenin have direct functions in tumor metastasis, and the overexpression of Sox 2 and $\beta$-catenin can stimulate EMT. Therefore, inhibition of $\beta$-catenin expression may have a role in inhibiting the EMT process and consequently repressing metastatic tumor spread.

Migratory cancer cells remodel their cell-cell and cellmatrix adhesions, which requires remodeling of the actin cytoskeleton $(54,55)$. Reorganization of the actin cytoskeleton is one of the essential mechanisms involved in the regulation of cell migration. Many factors promote actin reorganization and cell motility, including small GTPases, such as RhoA, Rho-associated protein kinase (ROCK) and Cdc42. These proteins cycle between active and inactive states by binding to GTP or GDP, respectively. With regard to the possible action mechanism, simvastatin blocks the geranylgeranylation of RhoA, thereby blocking the translocation of RhoA from the cytoplasm to the membrane, which is where it binds a set of effector molecules that are important for maintaining the undifferentiated status of the cell; thus, we can also speculate that the inhibition of RhoA function by simvastatin may induce differentiation-related processes. From another point of view, the inhibition of RhoA geranylation may account for the inhibition of ROCK2 activity, which has an important role in mediating structural changes in the actin cytoskeleton.

Another interesting aspect is the strain transfer from the cytoskeleton to the nucleus. Evidence indicates that force can be transmitted from the cytoskeleton to the nucleus, and that these mechanical stimuli, which act on specific mechanosensory complexes, are able to influence certain gene expression pathways. It has been shown that the YAP/TAZ and ERK1/2 pathways can be influenced by dynamic tensile loading, with consequent transcriptional regulation of specific genes involved in the proliferation/differentiation equilibrium. The inhibition of ROCK, which directly regulates actin polymerization and de-polymerization, decreases the nuclear strain transfer and leads to loss of the cytoskeletal-to-nucleoskeletal connectivity required for the transmission of regulatory signals. On the basis of these studies, inhibition of ROCK may induce the downregulation of stemness-related genes, which may be considered cytoskeleton- and nucleoskeletonassociated genes.

It has also been shown that RhoA controls the formation of stress fibers, while Cdc42 and Rac proteins influence the production of filopodia and lamellipodia (56). Various mechanisms or factors that influence the function of Rho appear to have fundamental roles in the regulation of cell motility and spread into the surrounding tissues; in this manner, RhoA has a pivotal role in the modulation of metastatic spread.

Simvastatin-mediated blocking of the prenylation and geranylgeranylation of Racl, Cd42 and RhoA inhibits the three principal pathways of actin polymerization and filopodia, lamellipodia and invadopodia formation; in this manner, simvastatin may decrease the capability of cancer cells to invade the extracellular matrix, and thus may have a potential role in the control of tumor progression.

In the present study, we demonstrated that simvastatin can inhibit normal actin polymerization (which is fundamental for cytoskeletal formation and maintenance of normal cytoarchitecture), thereby inducing the loss of actin cytoskeleton integrity and the relative inhibition of invadopodia formation, which can decrease cell motility and spreading in the extracellular matrix or other surrounding tissues; by these mechanisms, simvastatin may contribute to the reduction of metastatic spread. Furthermore, in NTERA-2 teratocarcinoma cells, HepG2 hepatoblastoma cells and MCF7 breast cancer cells, we demonstrated that simvastatin induces a strong downregulation of the three principal stemness genes, Oct 4 , Sox2, 
and Nanog, and inhibits the expression of $\beta$-catenin, which has a pivotal role in EMT.

Collectively, these data demonstrate that simvastatin, through inhibition of the mevalonate pathway and the associated inhibition of the farnesylation and geranylgeranylation processes, induces a destructuration of the cytoskeleton, and that it is the destabilized cytoskeleton that results in the inhibition of stemness gene expression and lamellipodia and filopodia formation. In this manner, simvastatin can modulate the equilibrium between proliferation, differentiation and metastatic spreading of cancer cells. On the basis of our experimental results, we believe that simvastatin, used at an appropriate dosage and in combination with conventional chemotherapy, may have a potential utility in the therapy or prevention of various cancer types.

\section{Materials and methods}

Cell culture. The NTERA-2 pluripotent human embryonic carcinoma cells, HepG2 human hepatoblastoma cells and MCF7 human breast cancer cells were purchased from the American Type Culture Collection (ATCC; Manassas, VA, USA) and cultured in Dulbecco's modified Eagle's medium (DMEM) supplemented with L-glutamine, $100 \mathrm{U} / \mathrm{ml}$ penicillin, $10 \mu \mathrm{g} / \mathrm{ml}$ streptomycin and $10 \%$ fetal bovine serum (FBS), in a $5 \% \mathrm{CO}_{2}$ incubator at $37^{\circ} \mathrm{C}$.

Simvastatin and cytochalasin treatment. Simvastatin (carboxylate form; Calbiochem-Merck Co., Darmstadt, Germany) was dissolved in dimethyl sulfoxide (DMSO) to produce a $20-\mathrm{mM}$ stock solution and stored at $-20^{\circ} \mathrm{C}$. In all experiments, plated cells were treated with $20 \mu \mathrm{M}$ simvastatin for $24 \mathrm{~h}$ in normal culture conditions. Cytochalasin D (C2618; Sigma-Aldrich, Hamburg, Germany) was prepared as $10-\mathrm{mM}$ stock solution in DMSO and used at concentration of $5 \mu \mathrm{M}$ in cell cultures for $6 \mathrm{~h}$. The control cells were treated with DMSO only.

RNA extraction and $c D N A$ synthesis. Total RNA was extracted from cells untreated or treated with simvastatin by scraping in TRIzol ${ }^{\circledR}$ reagent (Invitrogen, Carlsbad, CA, USA). Total RNA was purified according to the manufacturer's protocol and the purified RNA integrity was verified by electrophoresis on $1 \%$ agarose gel in $1 \mathrm{X}$ TBE. cDNA synthesis was carried out with an EasyScript ${ }^{\mathrm{TM}}$ cDNA Synthesis kit (Applied Biological Materials Inc., Richmond, BC, Canada) according to the manufacturer's protocol, starting from $1 \mu \mathrm{g}$ of total RNA. cDNA synthesis was verified by amplification of a housekeeping gene, such as $\beta$-actin or GAPDH (primers listed below), using the SapphireAmp ${ }^{\circledR}$ Fast PCR Master Mix, in an Applied Biosystems GeneAmp PCR System 9700 thermocycler.

Real-time quantitative PCR ( $q P C R$ ). The expression levels of stemness genes were quantified by qPCR. For each sample, a mixture consisting of $10 \mu \mathrm{l}$ of SYBR-Green (Thermo Scientific Maxima SYBR-Green/Fluorescein qPCR Master Mix 2X), $1 \mu \mathrm{l}$ reverse and forward primers $(10 \mu \mathrm{M})$, and $5 \mu \mathrm{l}$ cDNA was prepared in a $20 \mu \mathrm{l}$ of total volume, according to the manufacturer's recommendations, and analyzed in a Bio-Rad Thermal Cycler. The $\mathrm{Ct}$ values of the genes of interest were normalized with respect to the $\beta$-actin or GAPDH housekeeping gene. The
PCR conditions was: $10 \min 95^{\circ} \mathrm{C}$ for Taq activation, followed by 40 cycles of $15 \mathrm{sec}$ at $95^{\circ} \mathrm{C}$ for denaturation and $60 \mathrm{sec}$ at $60^{\circ} \mathrm{C}$ for annealing/extension.

The sequences of primers used were as follows: $h-\beta$-actin forward, GTGGCCGAGGACTTTGATTG and h- $\beta$-actin reverse, GGACTGGGCCATTCTCCTTA; GAPDH forward, AGTCAGCCG CATCTTCTTTT and GAPDH reverse, GTGA AGCGCCAGTGGACTC; h-OCT4 forward, ACATCAAAG C TCTGCAGAAAGAACT and h-OCT4 reverse, CTGAATAC CTTCCCAAATAGAACCC; h-SOX2 forward, TACAGCA TGTCCTACTCGA and h-SOX2 reverse, TGGAGTGGGA GGAAGAGGTA; h-NANOG forward, CAGCCAAATTCT CCTGCCAG and h-NANOG reverse, CACGTCTTCAGG TTGATGT; $h-\beta$-catenin forward, AGCTTCCAGACACGC TATCA and $h-\beta$-catenin reverse, CCAGTAAGCCCTCACG ATGA. All primers were purchased from Metabion International (Steinkirchen, Germany.

Immunofluorescence analysis. Untreated or simvastatintreated cells were grown on coverslips in 24-well cell culture plates to $\sim 80 \%$ confluence. In preparation for immunofluorescence, cells were rinsed with phosphate-buffered saline (PBS), fixed with $4 \%$ paraformaldehyde for $30 \mathrm{~min}$ at room temperature and permeabilized with $0.1 \%$ Triton $\mathrm{X}-100$ for $10 \mathrm{~min}$ at room temperature. The following primary antibodies were used: OCT-3/4 (C-10; \#sc-5279; 1:500; Santa Cruz Biotechnology, Inc., Santa Cruz, CA, USA); Sox2 (L1D6A2, cat. no. 4900; 1:500; Cell Signaling Technology, Danvers, MA, USA); Nanog (1E6C4, cat. no. 4893; 1:500; Cell Signaling Technology); and $\beta$-catenin (H-102; \#sc-7199; 1:500; Santa Cruz Biotechnology). After washing three times in PBS, cells were incubated with secondary antibodies (1:200; AlexaFluor; Molecular Probes/Thermo Fisher Scientific, Inc., Waltham, MA, USA). For actin detection, cells were stained with Alexa Fluor 488 Phalloidin (\#A12379); for the detection of cell membranes, cells were stained with the membrane stain wheat germ agglutinin-Alexa Fluor 488 conjugate (Invitrogen, Carlsbad, CA, USA) following the manufacturer's instructions. Cell nuclei were counterstained with DAPI (Invitrogen). The coverslips were mounted with a drop of Mowiol ${ }^{\circledR}$ mounting medium and observed with an AR1 confocal laser scanning microscope (Nikon, Tokyo, Japan) using the NIS elements software.

Wound-healing assay. In order to understand the effect of simvastatin on the progression and invasion of the cancer cells utilized in our experiments, we performed a woundhealing assay. In brief, cells were seeded into $60-\mathrm{mm}$ dishes at $5 \times 10^{5}$ cells/plate. When the confluence reached $\sim 90 \%$, a single scratch wound was created on the cell layer with a $200 \mu \mathrm{l}$ pipette tip. After $24 \mathrm{~h}$, in untreated cells and in $20 \mu \mathrm{M}$ simvastatin-treated cells, the number of cells that had grown into the scratch area was observed by inverted microscopy (DMI6000 Leica microscope; Leica Microsystems, Wetzlar, Germany). The cells were quantified by counting.

Statistical analysis. Each experiment was repeated three times. Results are expressed as the mean \pm SEM, and the effects were compared with untreated control cells. Paired t-tests were used to analyze the effects of simvastatin and cytochalasin. $\mathrm{P}<0.05$ 
A

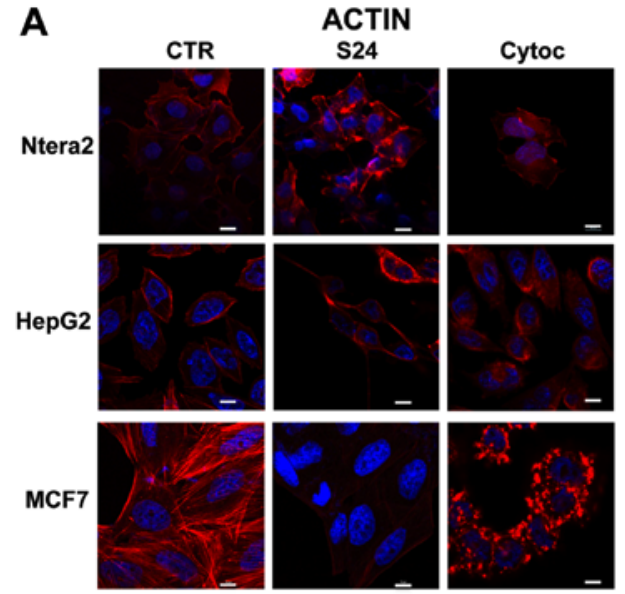

B

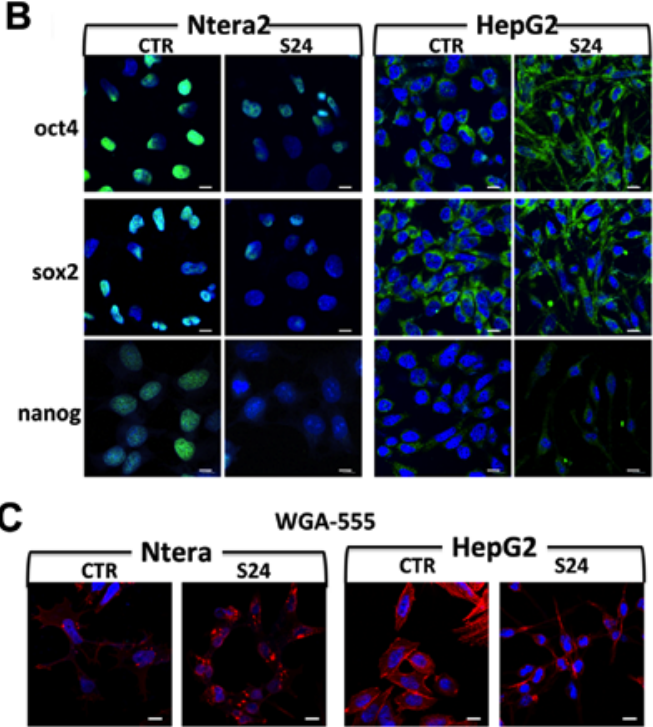

Figure 2. Confocal immunofluorescence analysis. The results of the immunofluorescence analysis of simvastatin- and cytochalasin-induced cytoskeletal modifications and stemness-related gene downregulation. (A) Merged images of phalloidin (red)/DAPI (blue) staining showing actin and cytoskeleton structural modifications induced by simvastatin and cytochalasin treatments in teratocarcinoma cells (NTERA-2), hepatoblastoma cells (HepG2) and breast cancer cells (MCF7). (B) Immunofluorescence analysis of Oct4, Sox 2 and Nanog (green)/DAPI (blue) (merged images), showing differences in expression induced by simvastatin in NTERA-2 and HepG2 cancer cells. More evident differences in the expression and localization of all three of the studied stemness-related genes were observed in NTERA-2 cells. (C) Simvastatin treatment for $24 \mathrm{~h}$ in HepG2 cells induced a very strong change in cell morphology, as shown by immunofluorescence analysis with cell membrane marker WGA555/DAPI (merged images). (CTR, control; S24, simvastatin 24 h; cytoc, cytochalasin 6 h; scale bar, $10 \mu \mathrm{m})$.

was considered to indicate statistical significance $(\mathrm{P}<0.05$; $\mathrm{P}<0.01 ; \mathrm{P}<0.001)$.

\section{Results}

Immunofluorescence analysis of the actin cytoskeleton. Treatment of NTERA-2, HepG2 and MCF7 human cancer cells with $20 \mu \mathrm{M}$ simvastatin for $24 \mathrm{~h}$ induced strong modifications in actin structure and cytoskeletal conformation (Fig. 2A). These modifications were different in the various cell lines, indicating a different sensitivity of each cell type to the drug. Moreover, simvastatin induced actin destructuration, which differed markedly from that observed following cytochalasin treatment (Fig. 2A).

In NTERA-2 cells, the cytoskeleton structural modification was obvious; in the untreated control cells, a well-structured frame of fibers occupied the entire cytoplasm and there were projections from the membrane cytoskeleton to the nucleus, whereas this organization had completely disappeared in the simvastatin-treated cells. The same result was obtained with the cytochalasin treatment. In both treatment groups, the actin molecules accumulated in a disorganized manner in parts of the cytoplasm.

In untreated HepG2 cells, the actin fibers were localized mainly at the periphery, inside the membrane, with well-defined projections to the nuclei. After the simvastatin treatment (Fig. 2A), this organization disappeared completely, and the actin remained localized at the extremities of the cell, and appeared compressed, assuming a polarized form. In presence of the cytochalasin, none of this structure was maintained, and the fibers were not visible, but the actin appeared to accumulate in a disorganized manner in the cytoplasm.
In untreated MCF7 breast cancer cells, the structure of the actin fibers was evident, with clear projections from the membrane to the nuclei. With simvastatin, this structure was gradually lost and the fiber bundles were less evident. The addition of cytochalasin induced a complete loss of the fiber conformation, and the actin appeared fragmented in the cytoplasm (Fig. 2A).

These results suggest that simvastatin induces cytoskeletal conformational changes, presumably related to the loss of post-translational modification of proteins involved in actin cytoskeleton formation, such as RhoA, Cd42 or ROCK GTPases. These proteins, to be functionally active, must be prenylated or geranylated, and simvastatin specifically inhibits these modifications.

Simvastatin induces changes in the localization of stemness-related proteins. The expression of the selected stemness-related proteins was studied by immunofluorescence analysis following simvastatin treatment.

As shown in Fig. 2B, stronger expression of the stemnessrelated proteins was observed in the NTERA-2 cells and, specifically in this cell population, the changes in the stemness protein localization in the presence of simvastatin were more clearly observable. In untreated NTERA-2 cells, the expression of Oct4 was very strong, and the protein was localized only in the nuclei. Simvastatin treatments significantly reduced the Oct4 protein expression in the nuclei (Fig. 2B). Similar results were obtained with Sox 2: the expression of the protein was very strong, with the protein concentrated principally in the nuclei, and the simvastatin treatment induced an evident decrease in the expression of Sox 2, with only a minority of nuclei showing expression of the protein. Similarly, the expression of Nanog was evident in the nuclei in untreated cells; by contrast, in cells 

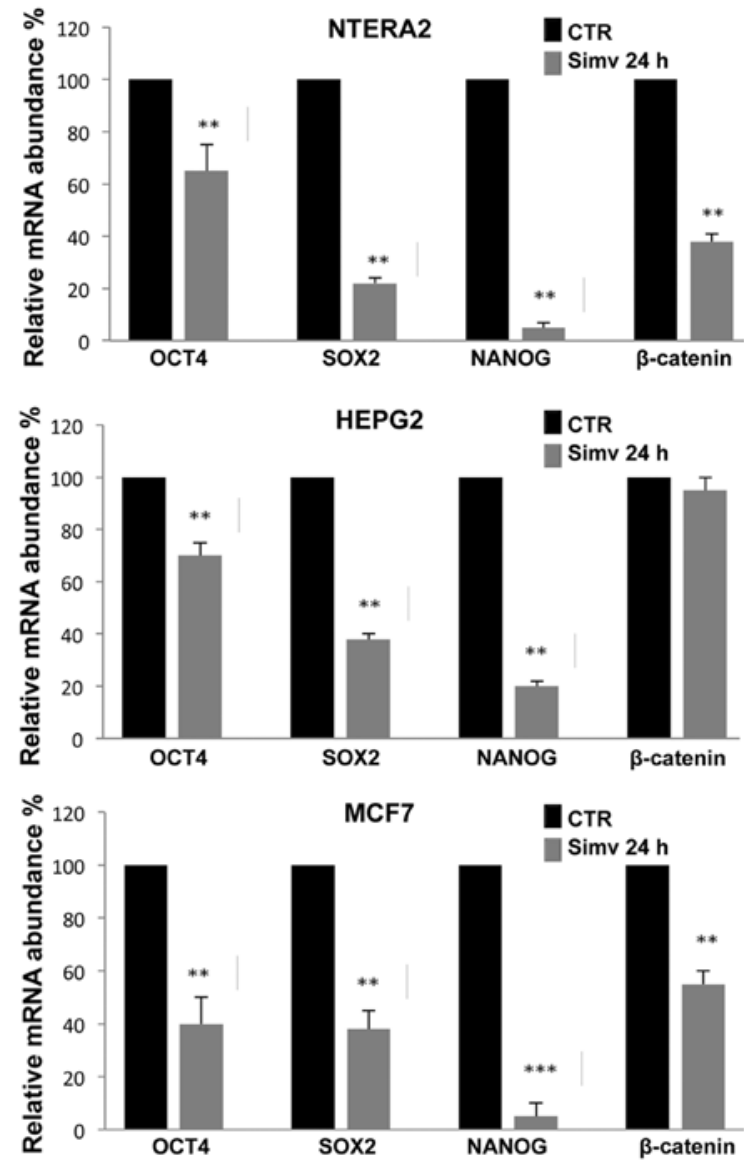

Figure 3. Simvastatin induces downregulation of stemness-related and $\beta$-catenin genes. A total of $20-\mu \mathrm{M}$ simvastatin treatment for $24 \mathrm{~h}$ significantly inhibited the expression of stemness-related genes in NTERA-2, HepG2 and MCF7 human cancer cells. Oct4, Sox 2 and Nanog expression levels were normalized based on control untreated cells (100\%); Oct4 levels were reduced by $40-60 \%$, while Sox 2 levels were reduced by $>60 \%$. The reduction of Nanog expression was much stronger, at $\sim 90 \%$ reduction. At the same time, the expression of the EMT marker $\beta$-catenin was reduced at a level comparable with that of Oct4 and Sox 2 in NTERA-2 and MCF7 cells, whereas minimal reduction of $\beta$-catenin was observed in HepG 2 cells. The results are presented as the mean \pm SD of three experiments, each performed in triplicate. ${ }^{* *} \mathrm{P}<0.01 ;{ }^{* * *} \mathrm{P}<0.001$.

treated with simvastatin, the protein expression was strongly reduced and almost disappeared from the nuclei (Fig. 2B).

Less evident were the localization differences obtained in untreated HepG2 cells. In these cells, the morphological changes induced by the simvastatin were very strong, and the cytoplasm was compacted at the two cell poles; for this reason, the Oct 4 and Sox 2 proteins, which in untreated cells appeared uniformly localized around the nucleus, were confined at the extremities of the cell. This particular form does allow individuation of the specific changes in stemness protein localization (Fig. 2B). Nanog is expressed at a low level in HepG2 cells, and the localization differences after simvastatin treatment were limited (Fig. 2B). These findings suggest that, in some cells, entry of the protein into the nucleus is impaired, and thus the protein remains accumulated in the cytoplasm.

In MCF7 cells, the levels of the stemness-related proteins were very low, and it was not possible to observe differences in expression and localization between the untreated and simvastatin-treated cells (data not shown).
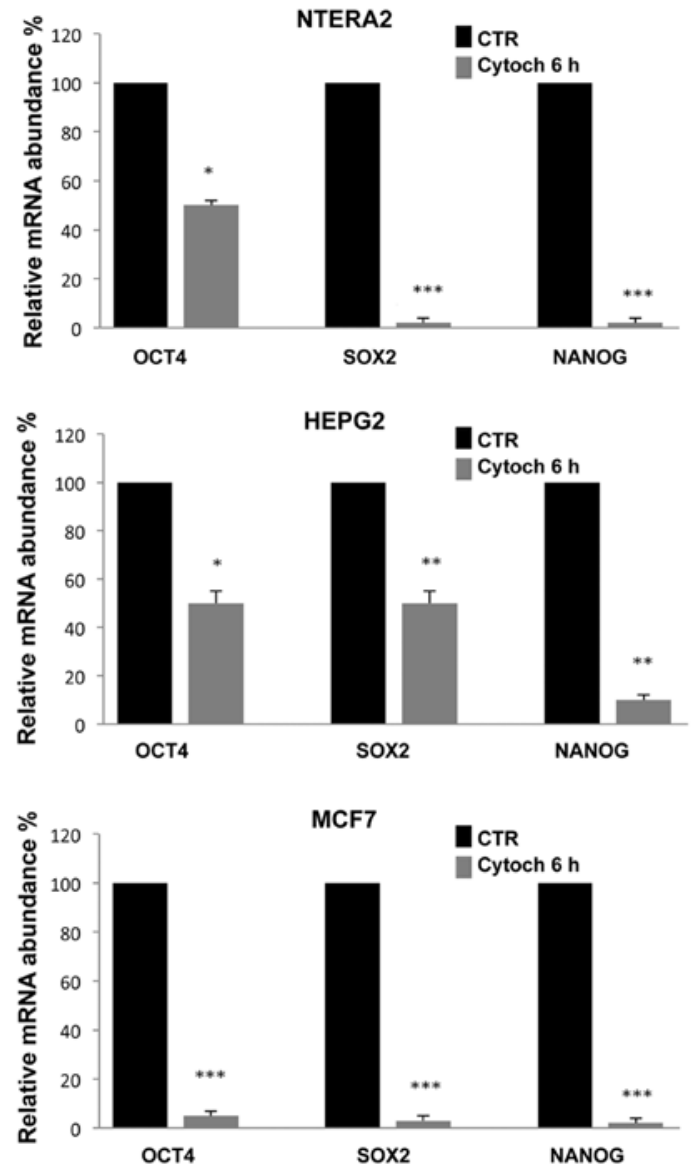

Figure 4. Cytochalasin reduces the expression of stemness-related genes. Treatment with $5 \mu \mathrm{M}$ cytochalasin for $6 \mathrm{~h}$ inhibited the expression of the stemness-related genes in NTERA-2, HepG2 and MCF7 human cancer cells more than simvastatin. Oct 4 expression, normalized to $100 \%$ in control untreated cells, was reduced by between 50 and $90 \%$. Sox 2 and Nanog expression levels were reduced by $90 \%$. The results are presented as the mean $\pm \mathrm{SD}$ of three experiments, each performed in triplicate. ${ }^{*} \mathrm{P}<0.05$; ${ }^{* *} \mathrm{P}<0.01 ;{ }^{* * *} \mathrm{P}<0.001$

As shown in Fig. 2C, in NTERA-2 and HepG2 cells, simvastatin induced morphological modifications; immunofluorescence analysis using the membrane marker WGA-488 showed the effects of simvastatin on cell morphology, as previously shown in Fig. 2A, wherein cells were marked with phalloidin to reveal actin structure.

Stemness-related gene expression in simvastatin- and cytochalasin-treated cells. As shown in Fig. 3, RT-qPCR analysis revealed that $20 \mu \mathrm{M}$ simvastatin treatment for $24 \mathrm{~h}$ strongly inhibited the expression of the stemness-related genes Oct4, Sox 2 and Nanog in the three human cancer cell lines investigated (NTERA-2, HepG2 and MCF7). In all three cell types analyzed, the effects of simvastatin treatment were most evident with Nanog expression, which in NTERA-2 and MCF7 reached an inhibition rate of $90 \%$. More limited was the expression reduction observed for Oct 4 , which was $\sim 60 \%$, in all the cells analyzed while for Sox 2 the greatest inhibition rate was observed in NTERA-2 cells. The reduction of $\beta$-catenin expression induced by simvastatin was evident only in NTERA-2 and MCF7 cells, while no substantial inhibition was observed in HepG2 cells (Fig. 3). 


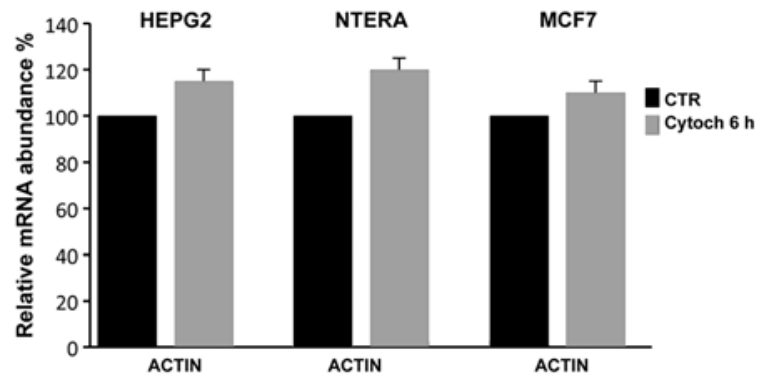

Figure 5. Cytochalasin does not influence actin expression. To verify whether cytochalasin treatment influenced the expression of actin, we controlled its expression relative to another house-keeping gene, GAPDH, demonstrating that cytochalasin did not inhibit actin expression.
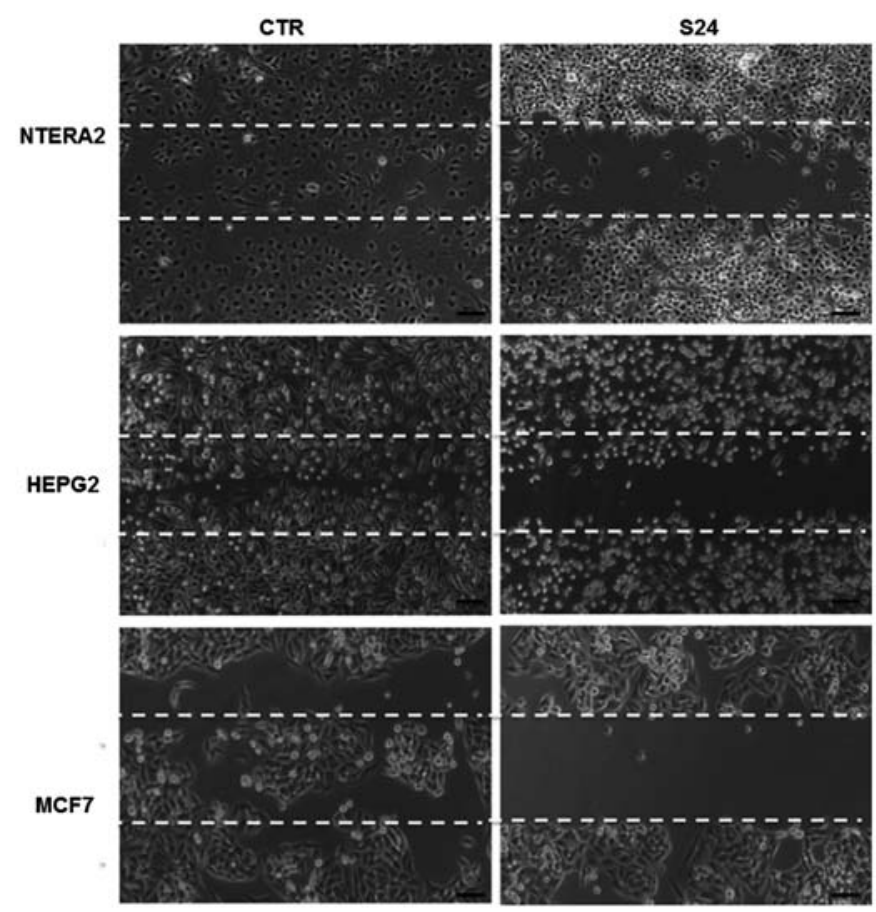

Figure 6. Wound healing analysis. The simvastatin treatment clearly inhibited the cell growth and migration of NTERA-2, HepG2 and MCF7 cells. In untreated control cells (CTR) after $24 \mathrm{~h}$, the scratched area appeared completely repopulated. By contrast, in simvastatin treated cells (S24), the scratched area remained unpopulated after $24 \mathrm{~h}$, indicating inhibition of migration. This experiment was replicated three times to confirm the results.

To evaluate whether the inhibition of stemness-related gene expression was correlated with the destructuration of the cytoskeleton, we cultured cells in the presence of $5 \mu \mathrm{M}$ cytochalasin, a well known inhibitor of actin polymerization. Notably, after 6 h of culture, the cytochalasin induced a significant reduction in the expression of all three stemness-related genes analyzed, which, as shown in Fig. 4, was comparable to the inhibition levels induced by simvastatin. The most evident reduction was observed in NTERA-2 and MCF7 cells, and the gene most sensitive to cytochalasin treatment was Nanog.

To verify whether the cytochalasin treatment affected the expression of actin, which is used in RT-qPCR expression analyses as an internal standard, we controlled its expression relative to another house-keeping gene, GAPDH; as shown in Fig. 5, even when actin polymerization and cell cytoskeletal structure were evidently inhibited, cytochalasin did not inhibit the actin expression, demonstrating that it could be used as an internal control in RT-qPCR expression analyses even in the presence of cytochalasin.

We hypothesized that alteration of the actin cytoskeleton structure may induce modification of the projections of actin fibers to the nucleoskeleton, and that modification of this strain transfer may have a regulatory action on certain stemnessrelated genes. RT-qPCR demonstrated that simvastatin and cytochalasin could downregulate the transcription of the analyzed stemness genes, and simvastatin treatment was found to induce a change in the expression and localization of the respective proteins.

Wound healing assay. To explore whether simvastatin treatment could affect cell migration, we performed a scratchwound assay in NTERA-2, HepG2 and MCF7 cells. As shown in Fig. 6, in the untreated cells, the scratched area was nearly completely repopulated after $24 \mathrm{~h}$; by contrast, in the cells treated with $20 \mu \mathrm{M}$ simvastatin, the cell growth was inhibited and the scratched area remained unpopulated after $24 \mathrm{~h}$, demonstrating that simvastatin may inhibit cancer cell growth and migration. This experiment was replicated three times, confirming the results obtained.

\section{Discussion}

In the present study, we demonstrated that simvastatin, by blocking the mevalonate pathway and the associated processes of farnesylation and geranylgeranylation, induces inhibition of the normal actin polymerization that is fundamental for cytoskeletal formation and maintenance of normal cytoarchitecture; the resultant destabilization of the cytoskeleton seems to inhibit the expression of stemness-related genes, which likely modulates the equilibrium between proliferation and differentiation.

Moreover, loss of actin cytoskeleton integrity inhibits lamellipodia and invadopodia formation, decreasing cell motility and invasion of the cancer cells into the extracellular matrix or surrounding tissues, thereby reducing metastatic spread. Inhibition of cell spreading by simvastatin was also demonstrated by wound healing experiments, in which simvastatin appeared to reduce cell migration.

From a more general perspective, we could hypothesize that any condition or drug that affects the structure of the cytoskeleton may influence the formation of actin fiber projections from the cytoskeleton to the nucleoskeleton. If actin projections to the nucleoskeleton exert a strain transfer and, as observed, this strain has regulatory action on certain genes, we can hypothesize the presence of mechanosensitive molecules at or within cell membranes confers to the cytoskeleton a more important function beyond simply its structural functions. In this scenario, active communication between the membrane cytoskeleton and the nucleoskeleton may occur, with regulatory message transfer, and thus, we may hypothesize the existence of a functional mechanotransduction pathway that depends specifically on nuclear strain transfer and nuclear deformation.

These findings may lead to a more dynamic perspective on cell-cell interactions within a tissue or an organ. Each 
individual cell, through partial perturbation of the membrane cytoskeleton, could be affected by the presence of neighboring cells, and changes in the conformational state, induced by changes in cell morphology, could lead to cryptic binding sites being exposed, thereby recruiting or activating molecules involved in downstream signaling (56).

In this complex interaction system, any perturbation at the membrane level could be transferred through the cytoskeleton to the nucleoskeleton of the neighboring cells. This strain transfer could exert regulatory pressure on target genes.

Collectively, these aspects could produce a directional flow of information by which, in different moments, different genes could be overexpressed or underexpressed in the three spatial directions. If the cytoskeleton exercises a function so important, it is easy to imagine that any destruction of the actin framework may induce a silencing or a strong downregulation of all the genes controlled by the transfer of the actomyosin fiber strain.

In our experiments, we demonstrated that simvastatin has the capability to strongly influence cytoskeletal conformation. It has been well-documented that simvastatin blocks the isoprenylation and geranylgeranylation of RhoA, which in turn blocks the translocation of RhoA from the cytoplasm to the membrane, where it can bind to a set of effector molecules that are important for maintaining the undifferentiated status of the cell. On this basis, we may also speculate that the simvastatin-induced inhibition of RhoA functioning may indirectly induce differentiation-related processes. Moreover, the inhibition of RhoA geranylation may account for the inhibition of ROCK activity, which has an important role in mediating changes in the structure of the actin cytoskeleton. Thus, simvastatin, by directly regulating actin polymerization and de-polymerization, can decrease strain transfer to the nucleus and induce loss of the cytoskeletalto-nucleoskeletal connectivity; this connectivity is required for the transmission of regulatory signals that activate kinase cascades or transcriptional factors controlling the activation of proliferative genes or inactivation of differentiation genes. The loss of these signals may induce the downregulation of certain stemness-related genes which, on the basis of these considerations, could be considered nucleoskeletonassociated and mechanically regulated genes. However, we think that is not easy to define if the de-structuration of the cytoskeleton is a secondary effect of simvastatin treatment or the inhibition of post-translational protein modification, have a precise role in the structuration of actin cytoskeleton, according to our hypothesis more than alteration of cytoskeleton is the alteration of the strain transfer to have a direct role in the transmission of regulatory signal on genes expression. We want, however, to underline that ours is not a conclusion, but this function of simvastatin is at the moment only a hypothesis and more experiments are necessary to confirm this possible regulatory mechanism.

If the target genes of these pathways are proliferative genes, as the stemness genes typically are, this regulatory action could lead to different cell proliferation rates in different spatial directions, and this could result in preferential localized cell growth, whereby exercising additional directional pressure could induce further directional growth, with possible feedback regulation.
On another more general point of view, it is easy to consider that differences in cell proliferation in the three spatial directions, in a determined time interval, form the basis of the determination of body shape. When this occurs during normal development, in the absence of a pathological condition, normal morphological differences result. Outside normal development, a directional or localized activation of cell proliferation can result in a pathological state and, if this hyper-proliferation derives from the activation of proliferative genes and the inhibition of differentiation genes, a tumor phenotype may occur.

Thus, it appears likely that perturbation or destruction of the cytoskeleton by simvastatin, resulting in inhibition of strain transfer, may have important effects on the control of pathological cell growth. We may also hypothesize that the cell membrane and cytoskeleton serve a much more general role, with a sort of additional code for the control of the gene activity. In this context, the membrane proteins could control the strain transfer from cytoskeleton to nucleus, which could have a role in the transcriptional control of stemness- and/or differentiation-related genes.

In the present study, we have described, in several cancer cell types, the role of simvastatin in inducing the destruction of the cytoskeleton and the resultant transcriptional inhibition of three well-studied stemness genes, Oct4, Sox 2 and Nanog. We obtained the same results, including the significant inhibition of expression of Oct4, Sox 2 and Nanog, with the use of cytochalasin D, a drug usually used to inhibit cytoskeleton formation.

On the basis of these results, we can speculate that the downregulation of stemness-related gene expression may be determined by the loss of appropriate strain transfer from cytoskeleton to nucleus, which does not allow the exposure of cryptic binding sites and recruitment of the molecules normally involved in the downstream regulatory cascade.

As mentioned previously, the effect of simvastatin derives from the inhibition of RhoA and ROCK GTPase activity, as consequence of the inhibition of the prenylation and geranylgeranylation of these proteins. These GTPases have an important effect on the regulation of the translation rate and exact localization of the protein within the cell.

We have observed, principally in NTERA-2 cells, in which Oct4, Sox 2 and Nanog genes are strongly expressed, a reduction in the nuclear localization of all three stemness-related proteins. The evident loss of localization of these proteins in the nuclei, where they function as important transcription factors, leads us to believe that simvastatin could have a possible role in the inactivation of the function of stemness genes, which may result in the blocking of proliferation and induction of differentiation. In the other analyzed cancer cell lines, the effect of simvastatin was less clear than in NTERA-2 cells, suggesting that the inhibitory effect of simvastatin predominantly occurs in cells in which stemness gene expression is strong.

Moreover, by blocking the prenylation and geranylgeranylation of Rac1 and Cd42, simvastatin inhibits the three principal pathways of actin polymerization that occur during the formation of protrusions during cell movement, as well as the translocation and retraction steps involved in filopodia, lamellipodia and invadopodia formation. In this manner, this drug may decrease the ability of cancer cells to invade the 
extracellular matrix. The capacity of cancer cells to move through the extracellular matrix and into neighboring tissues is the basis of the process of metastasis formation. We suggest that simvastatin, through inhibition of appropriate cytoskeleton formation, may suppress the formation of invadopodia complexes necessary for cancer cell invasion. On the basis of these considerations, we hypothesize that simvastatin, in combination with conventional therapy, may serve a possible role in the control of tumor progression.

\section{Acknowledgements}

The authors are grateful to Mr. F. Moscatiello and Mr. S. Arbucci for their skillful technical assistance. The present study was financially supported by the Institute of Genetics and Biophysics A. Buzzati Traverso CNR.

\section{References}

1. Spampanato C, De Maria S, Sarnataro M, Giordano E, Zanfardino M, Baiano S, Cartenì M and Morelli F: Simvastatin inhibits cancer cell growth by inducing apoptosis correlated to activation of Bax and down-regulation of BCL-2 gene expression. Int J Oncol 40: 935-941, 2012.

2. Zanfardino M, Spampanato C, De Cicco R, Buommino E, De Filippis A, Baiano S, Barra A and Morelli F: Simvastatin reduces melanoma progression in a murine model. Int $\mathrm{J}$ Oncol 43: 1763-1770, 2013

3. Liao JK and Laufs U: Pleiotropic effects of statins. Annu Rev Pharmacol Toxicol 45: 89-118, 2005.

4. Goldstein JL and Brown MS: Regulation of the mevalonate pathway. Nature 343: 425-430, 1990.

5. Lee MH, Cho YS and Han YM: Simvastatin suppresses selfrenewal of mouse embryonic stem cells by inhibiting RhoA geranylgeranylation. Stem Cells 25: 1654-1663, 2007.

6. Sorrentino G, Ruggeri N, Specchia V, Cordenonsi M, Mano M, Dupont S, Manfrin A, Ingallina E, Sommaggio R, Piazza S, et al: Metabolic control of YAP and TAZ by the mevalonate pathway. Nat Cell Biol 16: 357-366, 2014.

7. Zhang S and Cui W: Sox2, a key factor in the regulation of pluripotency and neural differentiation. World J Stem Cells 6: 305-311, 2014.

8. Boyer LA, Lee TI, Cole MF, Johnstone SE, Levine SS, Zucker JP, Guenther MG, Kumar RM, Murray HL, Jenner RG, et al: Core transcriptional regulatory circuitry in human embryonic stem cells. Cell 122: 947-956, 2005.

9. Chen X, Xu H, Yuan P, Fang F, Huss M, Vega VB, Wong E, Orlov YL, Zhang W, Jiang J, et al: Integration of external signaling pathways with the core transcriptional network in embryonic stem cells. Cell 133: 1106-1117, 2008.

10. Loh KM and Lim B: A precarious balance: Pluripotency factors as lineage specifiers. Cell Stem Cell 8: 363-369, 2011.

11. Thomson M, Liu SJ, Zou LN, Smith Z, Meissner A and Ramanathan S: Pluripotency factors in embryonic stem cells regulate differentiation into germ layers. Cell 145: 875-889, 2011.

12. Askarian-Amiri ME, Seyfoddin V, Smart CE, Wang J, Kim JE, Hansji H, Baguley BC, Finlay GJ and Leung EY: Emerging role of long non-coding RNA SOX2OT in SOX2 regulation in breast cancer. PLoS One 9: e102140, 2014.

13. Bowles J, Schepers G and Koopman P: Phylogeny of the SOX family of developmental transcription factors based on sequence and structural indicators. Dev Biol 227: 239-255, 2000.

14. Wilson M and Koopman P: Matching SOX: Partner proteins and co-factors of the SOX family of transcriptional regulators. Curr Opin Genet Dev 12: 441-446, 2002.

15. Kamachi Y, Uchikawa M and Kondoh H: Pairing SOX off: With partners in the regulation of embryonic development. Trends Genet 16: 182-187, 2000.

16. Ambrosetti DC, Schöler HR, Dailey L and Basilico C: Modulation of the activity of multiple transcriptional activation domains by the DNA binding domains mediates the synergistic action of Sox 2 and Oct- 3 on the fibroblast growth factor-4 enhancer. J Biol Chem 275: 23387-23397, 2000.
17. Loh YH, Wu Q, Chew JL, Vega VB, Zhang W, Chen X, Bourque G, George J, Leong B, Liu J, et al: The Oct4 and Nanog transcription network regulates pluripotency in mouse embryonic stem cells. Nat Genet 38: 431-440, 2006.

18. Tomioka M, Nishimoto M, Miyagi S, Katayanagi T, Fukui N, Niwa H, Muramatsu M and Okuda A: Identification of Sox-2 regulatory region which is under the control of Oct-3/4-Sox-2 complex. Nucleic Acids Res 30: 3202-3213, 2002.

19. Jauch R, Aksoy I, Hutchins AP, Ng CK, Tian XF, Chen J, Palasingam P, Robson P, Stanton LW and Kolatkar PR: Conversion of Sox17 into a pluripotency reprogramming factor by reengineering its association with Oct4 on DNA. Stem Cells 29: 940-951, 2011

20. Loh KM and Lim B: A precarious balance: Pluripotency factors as lineage specifiers. Cell Stem Cell 8: 363-369, 2011.

21. Wang Z, Oron E, Nelson B, Razis S and Ivanova N: Distinct lineage specification roles for NANOG, OCT4, and SOX2 in human embryonic stem cells. Cell Stem Cell 10: 440-454, 2012.

22. Takahashi $\mathrm{K}$ and Yamanaka S: Induction of pluripotent stem cells from mouse embryonic and adult fibroblast cultures by defined factors. Cell 126: 663-676, 2006.

23. Nakagawa M, Koyanagi M, Tanabe K, Takahashi K, Ichisaka T, Aoi T, Okita K, Mochiduki Y, Takizawa N and Yamanaka S: Generation of induced pluripotent stem cells without Myc from mouse and human fibroblasts. Nat Biotechnol 26: 101-106, 2008.

24. Huangfu D, Osafune K, Maehr R, Guo W, Eijkelenboom A, Chen S, Muhlestein W and Melton DA: Induction of pluripotent stem cells from primary human fibroblasts with only Oct 4 and Sox2. Nat Biotechnol 26: 1269-1275, 2008.

25. Okita K, Ichisaka T and Yamanaka S: Generation of germlinecompetent induced pluripotent stem cells. Nature 448: 313-317, 2007.

26. Alonso MM, Diez-Valle R, Manterola L, Rubio A, Liu D, Cortes-Santiago N, Urquiza L, Jauregi P, Lopez de Munain A, Sampron N, et al: Genetic and epigenetic modifications of Sox2 contribute to the invasive phenotype of malignant gliomas. PLoS One 6: e26740, 2011

27. Basu-Roy U, Seo E, Ramanathapuram L, Rapp TB, Perry JA, Orkin SH, Mansukhani A and Basilico C: Sox2 maintains self renewal of tumor-initiating cells in osteosarcomas. Oncogene 31: 2270-2282, 2012.

28. Chen Y, Shi L, Zhang L, Li R, Liang J, Yu W, Sun L, Yang X, Wang Y, Zhang Y, et al: The molecular mechanism governing the oncogenic potential of SOX2 in breast cancer. J Biol Chem 283: 17969-17978, 2008.

29. Cox JL, Wilder PJ, Desler M and Rizzino A: Elevating SOX2 levels deleteriously affects the growth of medulloblastoma and glioblastoma cells. PLoS One 7: e44087, 2012.

30. Girouard SD, Laga AC, Mihm MC, Scolyer RA, Thompson JF, Zhan Q, Widlund HR, Lee CW and Murphy GF: SOX2 contributes to melanoma cell invasion. Lab Invest 92: 362-370, 2012.

31. Leis O, Eguiara A, Lopez-Arribillaga E, Alberdi MJ, HernandezGarcia S, Elorriaga K, Pandiella A, Rezola R and Martin AG: Sox 2 expression in breast tumours and activation in breast cancer stem cells. Oncogene 31: 1354-1365, 2012.

32. Lengerke C, Fehm T, Kurth R, Neubauer H, Scheble V, Müller F, Schneider F, Petersen K, Wallwiener D, Kanz L, et al: Expression of the embryonic stem cell marker SOX2 in early-stage breast carcinoma. BMC Cancer 11: 42-52, 2011.

33. Li XL, Eishi Y, Bai YQ, Sakai H, Akiyama Y, Tani M, Takizawa T, Koike $M$ and Yuasa Y: Expression of the SRY-related HMG box protein SOX2 in human gastric carcinoma. Int J Oncol 24: 257-263, 2004.

34. Sanada Y, Yoshida K, Ohara M, Oeda M, Konishi K and Tsutani Y: Histopathologic evaluation of stepwise progression of pancreatic carcinoma with immunohistochemical analysis of gastric epithelial transcription factor SOX2: Comparison of expression patterns between invasive components and cancerous or nonneoplastic intraductal components. Pancreas 32: 164-170, 2006.

35. Sattler HP, Lensch R, Rohde V, Zimmer E, Meese E, Bonkhoff H, Retz M,Zwergel T, Bex A, Stoeckle M, et al: Novel amplification unit at chromosome 3q25-q27 in human prostate cancer. Prostate 45: 207-215, 2000.

36. Seo E, Basu-Roy U, Zavadil J, Basilico C and Mansukhani A: Distinct functions of Sox 2 control self-renewal and differentiation in the osteoblast lineage. Mol Cell Biol 31: 4593-4608, 2011. 
37. Ben-Porath I, Thomson MW, Carey VJ, Ge R, Bell GW, Regev A and Weinberg RA: An embryonic stem cell-like gene expression signature in poorly differentiated aggressive human tumors. Nat Genet 40: 499-507, 2008

38. Rodriguez-Pinilla SM, Sarrio D, Moreno-Bueno G, Rodriguez-Gil Y, Martinez MA, Hernandez L, Hardisson D, Reis-Filho JS and Palacios J: Sox2: A possible driver of the basal-like phenotype in sporadic breast cancer. Mod Pathol 20 : 474-481, 2007.

39. Lengerke C, Fehm T, Kurth R, Neubauer H, Scheble V, Müller F, Schneider F, Petersen K, Wallwiener D, Kanz L, et al: Expression of the embryonic stem cell marker SOX2 in early-stage breast carcinoma. BMC Cancer 11: 42-52, 2011.

40. Leis O, Eguiara A, Lopez-Arribillaga E, Alberdi MJ, HernandezGarcia S, Elorriaga K, Pandiella A, Rezola R and Martin AG: Sox 2 expression in breast tumours and activation in breast cancer stem cells. Oncogene 31: 1354-1365, 2012.

41. Basu-Roy U, Bayin NS, Rattanakorn K, Han E,Placantonakis DG, Mansukhani A and Basilico C: Sox2 antagonizes the Hippo pathway to maintain stemness in cancer cells. Nat Commun 6 $1-11,2015$.

42. Li X, Xu Y, Chen Y, Chen S, Jia X, Sun T, Liu Y, Li X, Xiang R and Li N: SOX2 promotes tumor metastasis by stimulating epithelial-to-mesenchymal transition via regulation of $\mathrm{WNT} / \beta$ catenin signal network. Cancer Lett 336: 379-389, 2013

43. Chen Y, Shi L, Zhang L, Li R, Liang J, Yu W, Sun L, Yang X, Wang Y,Zhang Y, et al: The molecular mechanism governing the oncogenic potential of SOX2 in breast cancer. J Biol Chem 283 $17969-17978,2008$.

44. Saigusa S, Tanaka K, Toiyama Y, Yokoe T, Okugawa Y, Ioue Y, Miki C and Kusunoki M: Correlation of CD133, OCT4, and SOX2 in rectal cancer and their association with distant recurrence after chemoradiotherapy. Ann Surg Oncol 16: 3488-3498, 2009.

45. Jia X, Li X, Xu Y, Zhang S, Mou W, Liu Y, Liu Y, Lv D, Liu CH, Tan X, et al: SOX2 promotes tumorigenesis and increases the anti-apoptotic property of human prostate cancer cell. J Mol Cell Biol 3: 230-238, 2011.
46. Polyak K and Weinberg RA: Transitions between epithelial and mesenchymal states: Acquisition of malignant and stem cell traits. Nat Rev Cancer 9: 265-273, 2009.

47. Xu J, Lamouille S and Derynck R: TGF-beta-induced epithelial to mesenchymal transition. Cell Res 19: 156-172, 2009.

48. Bryant DM and Stow JL: The ins and outs of E-cadherin trafficking. Trends Cell Biol 14: 427-434, 2004.

49. Ikenouchi J, Matsuda M, Furuse M and Tsukita S: Regulation of tight junctions during the epithelium-mesenchyme transition: Direct repression of the gene expression of claudins/occludin by Snail. J Cell Sci 116: 1959-1967, 2003.

50. Strauss R, Li ZY, Liu Y, Beyer I, Persson J, Sova P, Möller T, Pesonen S, Hemminki A, Hamerlik P, et al: Analysis of epithelial and mesenchymal markers in ovarian cancer reveals phenotypic heterogeneity and plasticity. PLoS One 6: e16186, 2011.

51. Jiang YG, Luo Y, He DL, Li X, Zhang LL, Peng T, Li MC and Lin YH: Role of Wnt/beta-catenin signaling pathway in epithelial-mesenchymal transition of human prostate cancer induced by hypoxia-inducible factor-1alpha. Int J Urol 14: 1034-1039, 2007.

52. Beavon IR: The E-cadherin-catenin complex in tumour metastasis: Structure, function and regulation. Eur J Cancer 36: $1607-1620,2000$

53. Brabletz T, Jung A, Spaderna S, Hlubek F and Kirchner T: Opinion: Migrating cancer stem cells - an integrated concept of malignant tumor progression. Nat Rev Cancer 5: 744-749, 2005.

54. Driscoll TP, Cosgrove BD, Heo SJ, Shurden ZE and Mauck RL: Cytoskeletal to nuclear strain transfer regulates YAP signaling in mesenchymal stem cells. Biophys J 108: 2783-2793, 2015.

55. Artman L, Dormoy-Raclet V, von Roretz C and Gallouzi IE: Planning your every move: The role of $\beta$-actin and its posttranscriptional regulation in cell motility. Semin Cell Dev Biol 34: 33-43, 2014.

56. Driscoll TP, Cosgrove BD, Heo SJ, Shurden ZE and Mauck RL: Cytoskeletal to nuclear transfer regulate YAP signaling in mesenchymal stem cells. Biophys J 108: 2783-2793, 2015. 